\title{
Self-Cleaning Antireflective Optical Coatings
}

\author{
Stefan Guldin, ${ }^{\dagger, \perp}$ Peter Kohn, ${ }^{\dagger}$ Morgan Stefik, $^{\text {gI }}$ Juho Song, ${ }^{\ddagger}$ Giorgio Divitini, ${ }^{\S}$ Fanny Ecarla," \\ Caterina Ducati, ${ }^{\S}$ Ulrich Wiesner, ${ }^{\ddagger}$ and Ullrich Steiner*, ${ }^{\dagger}$ \\ ${ }^{\dagger}$ Department of Physics, University of Cambridge, J J Thomson Avenue, Cambridge CB3 0HE, United Kingdom \\ ${ }^{\ddagger}$ Department of Materials Science and Engineering, Cornell University, Ithaca, New York 14853, United States \\ ${ }^{\S}$ Department of Materials Science and Metallurgy, University of Cambridge, Charles Babbage Road, Cambridge CB3 0FS, United \\ Kingdom \\ ${ }^{\|}$CSM Instruments, Rue de la Gare, 2034 Peseux, Switzerland

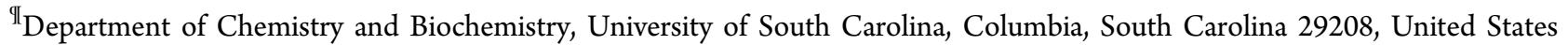

Supporting Information

ABSTRACT: Low-cost antireflection coatings (ARCs) on large optical surfaces are an ingredient-technology for high-performance solar cells. While nanoporous thin films that meet the zero-reflectance conditions on transparent substrates can be cheaply manufactured, their suitability for outdoor applications is limited by the lack of robustness and cleanability. Here, we present a simple method for the manufacture of robust selfcleaning ARCs. Our strategy relies on the self-assembly of a blockcopolymer in combination with silica-based sol-gel chemistry and preformed $\mathrm{TiO}_{2}$ nanocrystals. The spontaneous dense packing of copolymer micelles followed by a condensation reaction results in an inverse opal-type silica morphology that is loaded with $\mathrm{TiO}_{2}$ photocatalytic hot-spots. The very low volume fraction of the inorganic network allows the optimization of the antireflecting properties of the porous ARC despite the high refractive index of the embedded photocatalytic $\mathrm{TiO}_{2}$ nanocrystals. The resulting ARCs combine high optical and self-cleaning performance and can be deposited onto flexible plastic substrates.

KEYWORDS: Antireflective coatings, antireflection, self-cleaning, optical coatings, photocatalysis

$\mathrm{T}$ he reduction of light reflected off surfaces by antireflection coatings (ARCs) is well established and routinely used for eye glasses and high-performance optics. It typically relies on the tuning of thickness and refractive index of an ARC so that light reflecting off the two film surfaces interferes destructively, schematically shown in Figure $1 \mathrm{~b}$. This involves two wellknown principles: (1) For a given wavelength $\lambda$ and angle of incidence, the required optical thickness of the ARC must be $\lambda$ / 4 , and (2) the amplitude matching of the two reflected beams requires an effective refractive index of the $\operatorname{ARC}\left(n_{\mathrm{AR}}\right)$, which is the square-root of that of the optical substrate. ${ }^{1}$ For most optical substrates with refractive indices of around 1.5, the latter requirement implies unrealistically low values of $n_{\mathrm{ar}} \approx 1.22$. This conundrum is often solved by introducing suboptical porosity into the ARC. The effective refractive index is then a direct consequence of the material-air composite and can be approximated by effective medium theories such as the Bruggeman model. ${ }^{2,3}$ Mesoporous optical coatings have been realized by polymer phase separation on the sub-100 nm scale with subsequent removal of one of the phases. ${ }^{4,5}$ Other solution-based methods include the fabrication of nanoparticle films by random dense packing ${ }^{6}$ or electrostatic attraction, ${ }^{7}$ as well as the controlled assembly of charged colloids, ${ }^{8}$ hollow ${ }^{9}$ or mesoporous $^{10}$ silica spheres or polyelectrolyte multilayers. ${ }^{11}$ Vacuum-based fabrication approaches are typically relying on glancing angle chemical vapor deposition. ${ }^{12}$
An alternative concept to antireflection takes inspiration from the nanostructured topography of the corneal lenses found in moth eyes. ${ }^{13}$ As the structural features of the hexagonally arranged pattern of conelike protuberances are below the diffraction limit, the surface appears to have a continuous refractive index gradient between air and substrate that practically removes the optical interface. This was first realized in a photoresist structure by lithographical means. ${ }^{14}$ Recently, similar structures have been achieved on glass substrates by nanoimprint lithography ${ }^{15}$ as well as reactive ion etching with metal-particle ${ }^{16}$ or colloidal-based ${ }^{17}$ photolithographic masks. Although the optical principle is auspicious, experimental realizations are elaborate and currently can not compete with interference based approaches. ${ }^{18}$

While many experimental approaches have yielded researchgrade nanostructured ARCs that are close to perfection, ${ }^{19,20}$ their commercial implementation is hampered by their lack of robustness and the optical variability caused by contamination. Overcoming these two problems unfortunately leads to opposing requirements. Tough transparent materials (such as oxides and fluorides) have high surface energies giving rise to rapid pore filling by ambient organic contaminants, thus

Received: July 29, 2013

Revised: September 10, 2013

Published: October 14, 2013 


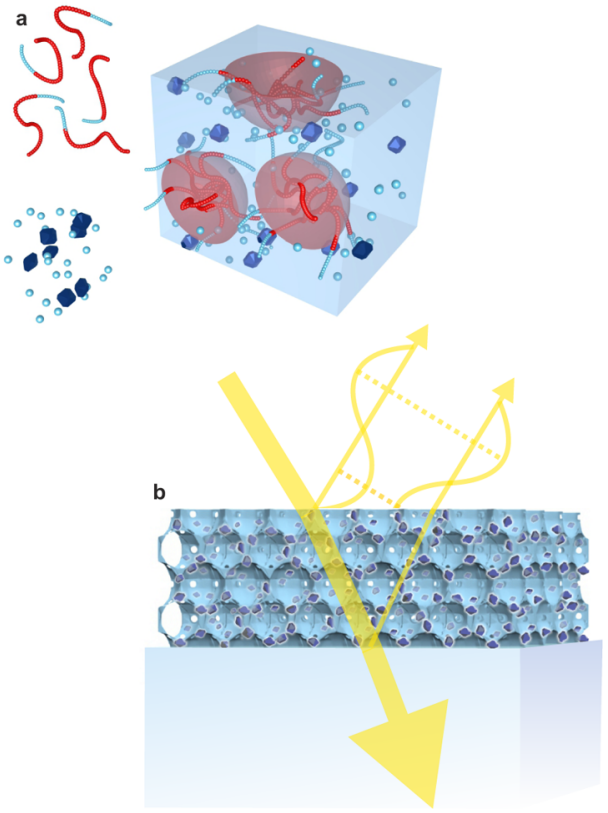

Figure 1. Schematic of ARC manufacture. (a) A solution of PI- $b$-PEO block copolymer, silica-based sol, and compatibilized $\mathrm{TiO}_{2}$ nanocrystals is used for the coating of transparent substrates. The inorganic components preferentially reside in the PEO phase and are therefore structure-directed during the micellar packing of the block copolymer. (b) Subsequent reactive ion-etching in an oxygen plasma removes the polymer and reveals an inorganic inverse opal-type structure that is loaded with $\mathrm{TiO}_{2}$ nanocrystals. Phase and amplitude matching of the optical coating give rise to destructive interference of reflected light, that is, enhanced transmission, while the embedded photocatalytic $\mathrm{TiO}_{2}$ nanocrystals prevent contamination of the ARC.

causing a deterioration of the AR effect. Porous low surfaceenergy organics, on the other hand, are mechanically fragile, and organic monolayers covering inorganic networks are not UV-stable.

Low-surface energy or superhydrophobic surfaces are selfcleaning in a sense that particulate contaminants adhere only very weakly and are easily washed off by water. The most commonly used route to introduce superhydrophobicity in ARCs is through silanization of the respective inorganic nanostructured coating. ${ }^{21-23}$ Other approaches include the use of organically modified silica ${ }^{24,25}$ or a postdeposition of PTFE. $^{26}$

On the contrary, photocatalytic ARCs do not rely on a cleaning medium, but decompose organic contaminants by light induced redox-reactions. ${ }^{27}$ While photocatalytic selfcleaning is in principle more robust and durable, the inclusion of a photocatalytic component in ARCs, typically $\mathrm{TiO}_{2}$, poses a major challenge because of its high refractive index $\left(n_{\mathrm{TiO}_{2}}>\right.$ $\left.2.5^{28}\right)$. Concepts to overcome the conflicting requirements for low refractive index and high photocatalytic activity include the surface coating of a colloidal-based ARC with $\mathrm{TiO}_{2}{ }^{29}$ the codeposition of $\mathrm{TiO}_{2}$ and $\mathrm{SiO}_{2}$ nanoparticles to form a porous ARC, ${ }^{7}$ the synthesis of $\mathrm{SiO}_{2} / \mathrm{TiO}_{2}$ core-shell particles, ${ }^{30}$ as well as a double layer structure of low refractive index $\mathrm{SiO}_{2}$ and $\mathrm{TiO}_{2}{ }^{31-33}$ All of these approaches achieve the required porosity with $n_{\mathrm{ar}} \approx 1.22$ only for very low $\mathrm{TiO}_{2}$ loading and require processing temperatures of above $400{ }^{\circ} \mathrm{C}$, which prevents their use on transparent plastics.
Here, we present a concept that combines antireflection and photocatalysis. It relies on the manufacture of a robust highly porous silica-based network with an effective refractive index much below the required value of around 1.22. This enables the incorporation of $\mathrm{TiO}_{2}$ nanocrystals into the network to an extent that restores $n_{\text {ar }}$ to its optimum. Furthermore, working with preformed $\mathrm{TiO}_{2}$ nanocrystals removes the requirement for high-temperature treatments to crystallize the oxide. Our method makes use of a high-molecular weight poly(isopreneblock-ethylene oxide) (PI-b-PEO) block copolymer (BCP) in combination with silica-based sol-gel chemistry and anatase $\mathrm{TiO}_{2}$ nanocrystals. In the right solvent, here in an azeotrope of toluene and 1-butanol, PI-b-PEO forms micelles with PI-cores in solution, which gives rise to a nanoscopic inverse opal-type structure of densely packed pores in a silica/ $/ \mathrm{TiO}_{2}$ matrix after solvent evaporation, sol-gel condensation, and removal of the polymer host. Robust continuous inorganic films of aluminosilica (referred to here as "silica") with $n_{\text {ar }}$ as low as 1.13 were obtained, allowing the incorporation of up to $50 \mathrm{wt} \%$ photocatalytic $\mathrm{TiO}_{2}$ nanocrystals while maintaining optimal AR properties. The resulting films have excellent photocatalytic properties and, because of their low processing temperatures, can be deployed on flexible plastic substrates.

Figure 1a illustrates the processing route for ARC manufacture. A high molecular weight PI-b-PEO BCP was dissolved in an azeotrope mixture of toluene and 1-butanol. Two different PI- $b$-PEO BCPs were used: BCP-34 (molecular weight $M_{\mathrm{n}}=34.4 \mathrm{~kg} \mathrm{~mol}^{-1}, 28$ wt $\left.\% \mathrm{PEO}, \mathrm{PDI}=1.05\right)$, and BCP-92 $\left(M_{\mathrm{n}}=91.9 \mathrm{~kg} \mathrm{~mol}^{-1}, 31.2 \mathrm{wt} \%\right.$ PEO, PDI = 1.09). A sol was prepared separately by the stepwise hydrolysis of a silicon/aluminum alkoxide mix. ${ }^{34,35} \mathrm{TiO}_{2}$ nanocrystals were synthesized via a nonhydrolytic sol-gel chemistry route ${ }^{36}$ which was modified to compatibilize the nanocrystals with the solvent mixture used to dissolve the polymer and sol. ${ }^{37}$ The components were subsequently combined with varying amounts of $\mathrm{TiO}_{2}$, which led to a hybrid solution that was processed into a thin film. Following temperature annealing to condense the inorganic matrix, the organic components were removed by reactive ion etching in an oxygen plasma. See Supporting Information for full experimental details.

Figure 2 shows the resulting porous inorganic films. The network morphologies in the scanning electron micrographs reveal their likely origin. The well-defined pore size and the local hexagonal arrangement are reminiscent of an inverse opaltype structure. The evolution of this morphology probably involved the formation of BCP micelles in solution, which during solvent evaporation self-assemble into an opal-type morphology consisting of a PI core and a sol-containing PEO matrix. The condensation reaction and polymer removal then gave rise to the porous network structure shown in Figure 2. In related studies on $\mathrm{PI}-b$-PEO assembled $\mathrm{TiO}_{2}$ networks, we have recently shown that pore interconnectivity can extend to micrometer thicknesses. ${ }^{38,39}$

Since the micellar size is determined by the polymer architecture, a variation of the organic-to-inorganic volume (or weight) fraction allows fine-tuning the porosity, while affecting the pore size only very little. The effect of a variation of the mixing ratio on porosity is shown in Figure $2 \mathrm{a}-\mathrm{c}$, where the polymer loading was increased from 28 to $50 \mathrm{wt} \%$. The weight percentages are defined as the BCP weight fraction relative to the total weight of utilized polymer and resulting silica-type material. 




Figure 2. Film morphology of mesoporous optical coatings. $(a-c)$ Scanning electron microscopy top-views of mesoporous silica films, stemming from a BCP solution with $M_{\mathrm{n}}=34.4 \mathrm{~kg} / \mathrm{mol}$ (BCP-34) and a polymer weight fraction of (a) $28 \%$, (b) $40 \%$, and (c) $50 \%$. (d) The use of a higher molecular weight polymer BCP of $M_{\mathrm{n}}=91.9 \mathrm{~kg} / \mathrm{mol}$ (BCP-92) results in significantly larger pores and identical refractive index for otherwise similar conditions ( $50 \%$ polymer weight content). The scale bars represent $100 \mathrm{~nm}$. (e) Variation of the resulting refractive index $(\lambda=632 \mathrm{~nm})$ for mesoporous films derived from BCP34 and BCP-92.

The pore size of the inorganic network can be separately controlled by varying the molecular weight of the sacrificial PI block. Figure $2 \mathrm{~d}$ shows the morphology of the film with similar inorganic loading as in Figure $2 \mathrm{c}$ but an increased molecular weight of the PI block. While a PI molecular weight of $24.8 \mathrm{~kg} /$ $\mathrm{mol}$ in BCP-34 led to a pore size of $\sim 33 \mathrm{~nm}$ (Figure 2a-c), increasing the PI block to $63.2 \mathrm{~kg} / \mathrm{mol}$ (BCP-92) resulted in $\sim 53 \mathrm{~nm}$-wide pores. This is in good agreement with scaling laws governing the radius of gyration of polymer chains in a good solvent. ${ }^{40}$ The pore interconnectivity arising from the close-packing of sacrificial micelles can be clearly identified. See also Figure S1 in the Supporting Information.

Spectroscopic ellipsometry of the resulting films shown in Figure $2 \mathrm{e}$, reveals the consequence of the variation in porosity of Figure $2 \mathrm{a}-\mathrm{d}$. Adjusting the polymer weight fraction in the initial solution from 28 to $67 \%$ resulted in a refractive index range of $1.40>n_{\mathrm{ar}}>1.13(\lambda=632 \mathrm{~nm})$. Following the Bruggeman effective medium approximation, a quasi-linear relationship between the polymer weight fraction, the resulting pore volume and hence the corresponding refractive index is expected. ${ }^{3}$ While the measured refractive index scales nearly linearly up to polymer content of $\approx 50 \mathrm{wt} \%, n_{\mathrm{ar}}$ levels-off for high organic contents. This is probably related to progressive shrinkage of the film with increasing polymer loading. An organic weight fraction of $50 \%$ effectively corresponds to a polymer volume fraction of $\sim 67 \%$. At these high polymer volume fractions, a certain densification of the inorganic network during processing and polymer removal is likely. Despite this apparent compaction, an increase in the polymer volume fraction up to $66 \%$ led to a continuous decrease in the film refractive index down to $n_{\mathrm{ar}}=1.13$

The lowest obtained refractive index $n_{\mathrm{ar}}=1.13$ for a silica network $(n=1.52)$ corresponds to a porosity of $73 \%$. In conventional approaches, porosity arises from the interstitial space between high refractive index components, such as silica nanoparticles or colloids. The jamming of monodisperse spheres limits the achievable porosity to $36-38 \% .{ }^{41}$ A further increase in pore volume can be realized by the introduction of a sacrificial spacer material with reported values of $50-60 \%{ }^{42}$ Using the present route, porosity generation is different since the pores arise from sacrificial micelles and not from the close packing of high refractive index components. The inorganic material resides in the hydrophilic outer layer of the micelles and is coassembled in an inverse opal-like geometry. The morphology therefore represents the densest packing of spherical pores, which allowed us to reach porosities as high as $73 \%$ after BCP removal. In contrast to a previous BCP route yielding porosities up to $66 \%,{ }^{43}$ our method is robust, scalable, and allows precise adjustment of pore size and concentration by controlling the place-holder micelles formed in solution.

Carefully tuning the refractive index to $n_{\mathrm{ar}}=1.22$ and the film thickness to $110 \mathrm{~nm}$ resulted in ARCs of high optical quality with light transmittance $T$ up to $99.9 \%$ at $540 \mathrm{~nm}$ (see Figure 4a). The transmission spectrum is very similar to that of a polymeric adhesive ARC named "Arktop" (Asahi Glass), the current benchmark, which achieved a similarly high peak value of $T_{\max }=99.5 \%$ but in a more limited spectral range (Figure S4 in the Supporting Information).

Adsorption of organic contaminants from the ambient atmosphere typically deteriorates the performance and longterm usability of mesoporous ARCs, particularly in outdoor applications. $\mathrm{TiO}_{2}$-based photocatalysis can be used to degrade the adsorbed hydrocarbons and restore the antireflective properties. In several previous studies, the amount of $\mathrm{TiO}_{2}$ that could be added to the ARC was small because of the resulting increase in refractive index. ${ }^{7,29,31,33}$

To improve the photocatalytic efficiency, we make use of the exceptionally low refractive index of our ARCs to maximize titania loading. Crystalline $4 \mathrm{~nm}$ sized $\mathrm{TiO}_{2}$ nanocrystals were synthesized using a nonhydrolytic sol-gel chemistry route ${ }^{36,37}$ and added to the sol solution. The direct incorporation of crystalline $\mathrm{TiO}_{2}$ from solution has two advantages: (1) no hightemperature step during film deposition is necessary to crystallize the $\mathrm{TiO}_{2}$, and (2) well-defined photoactive $\mathrm{TiO}_{2}$ hot-spots are formed within the network. While a posttreatment of ARCs with $\mathrm{TiO}_{2}$ precursor results in additional deposition of material, the present route enables $\mathrm{TiO}_{2}$ incorporation into the porous network itself.

The distribution of the nanocrystals within the mesoporous network was studied by transmission electron microscopy. The images presented in Figure $3 \mathrm{a}, \mathrm{b}$ of two different pore morphologies show well-dispersed 3-4 nm-wide nanocrystals, and no aggregates were detected. This is supported by a Scherrer analysis of the X-ray diffraction [101] anatase peak, 


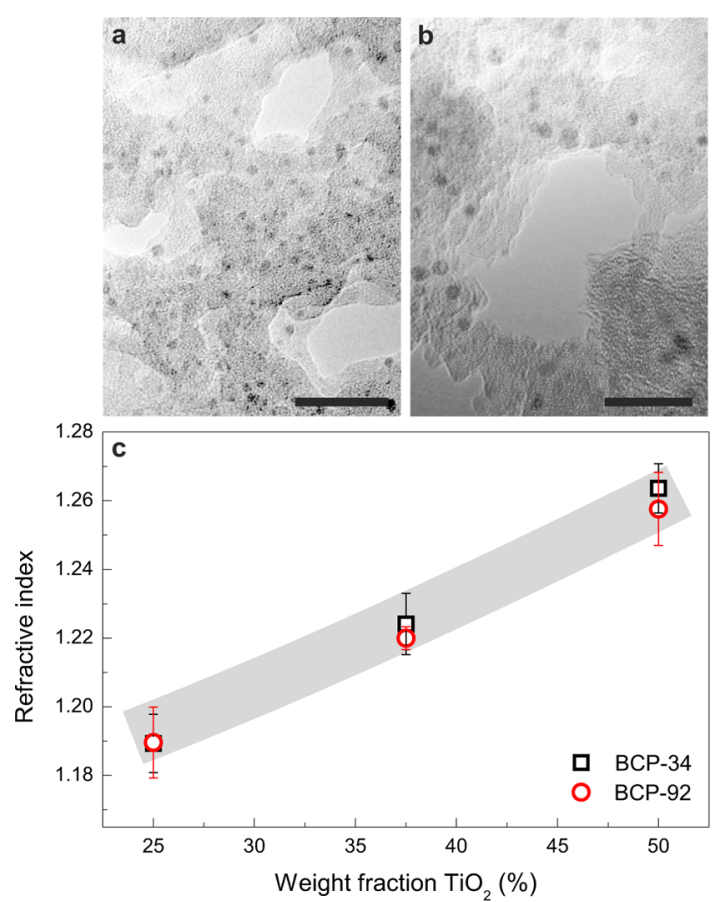

Figure 3. Composition of self-cleaning ARCs. High-resolution transmission electron micrographs showing the distribution of photocatalytic $\mathrm{TiO}_{2}$ nanocrystals embedded in the inorganic network for (a) BCP-34 and (b) BCP-92 derived films. The scale bars represent $20 \mathrm{~nm}$. (c) Refractive index as a function of $\mathrm{TiO}_{2}$ nanocrystal loading for BCP-34 and BCP-92 derived inorganic networks.

giving a crystal size of $3.5 \pm 0.2 \mathrm{~nm}$ (see Figure S2 in the Supporting Information).

Figure $3 \mathrm{c}$ shows the variation of the ARC refractive index as a function of the weight fraction of $\mathrm{TiO}_{2}$ nanocrystals in the initial hybrid solution $(\lambda=632 \mathrm{~nm})$. The increase in refractive index scales with $\mathrm{TiO}_{2}$ content and is in agreement with the Bruggeman effective medium approximation. ${ }^{2,3}$ Starting from a refractive index of $\sim 1.13$ for a neat mesoporous silica network, $n_{\mathrm{ar}}$ increased to $1.19,1.22$, and 1.26 for $\mathrm{TiO}_{2}$ weight fractions of 25.0, 37.5, and $50.0 \%$ in the initial solution. Thus, because of the $\sim 73 \%$ porosity of the inorganic network, up to $50 \mathrm{wt} \%$ aluminosilicate can be substituted by high refractive index $\mathrm{TiO}_{2}$ while achieving sufficiently low refractive indices for optimized ARCs.

The optical properties of the fabricated photocatalytic ARCs based on BCP-34 are shown in Figure 4. Substituting 50 wt \% aluminosilicate by photocatalytic $\mathrm{TiO}_{2}$ nanocrystals results in $T_{\max }=99.3 \%$ through a glass slide coated on both sides with an ARC (Figure 4a). The low-temperature ARC protocol enables the coating of flexible plastic substrates such as PET (polyethylene terephthalate), typically used in organic solar cells and flexible flat panel displays. ${ }^{44}$ In Figure $4 \mathrm{~b}$, we present the reflectance of a PET foil that was AR-coated on one side (PET-1s) as well as an uncoated reference (PET-0s). The reflectance of an uncoated $(A)$ and a coated PET interface $(B)$ follow from $R_{\mathrm{PET}-0 \mathrm{~s}}=A+((1-A) \times A)$ and $R_{\mathrm{PET}-1 \mathrm{~s}}=B+((1$ $-B) \times A$ ). The spectral traces are indicative of an ideal broadband antireflective response with a reduction in reflectance of up to $\sim 5.2 \%$ for each coated surface, resulting in reflectivities as low as $0.2 \%$. The high optical quality of the ARCs is also translated into near optimum transmittance of PET with singlesided coating, shown in Figure $\mathrm{S} 4 \mathrm{~b}$ in the Supporting
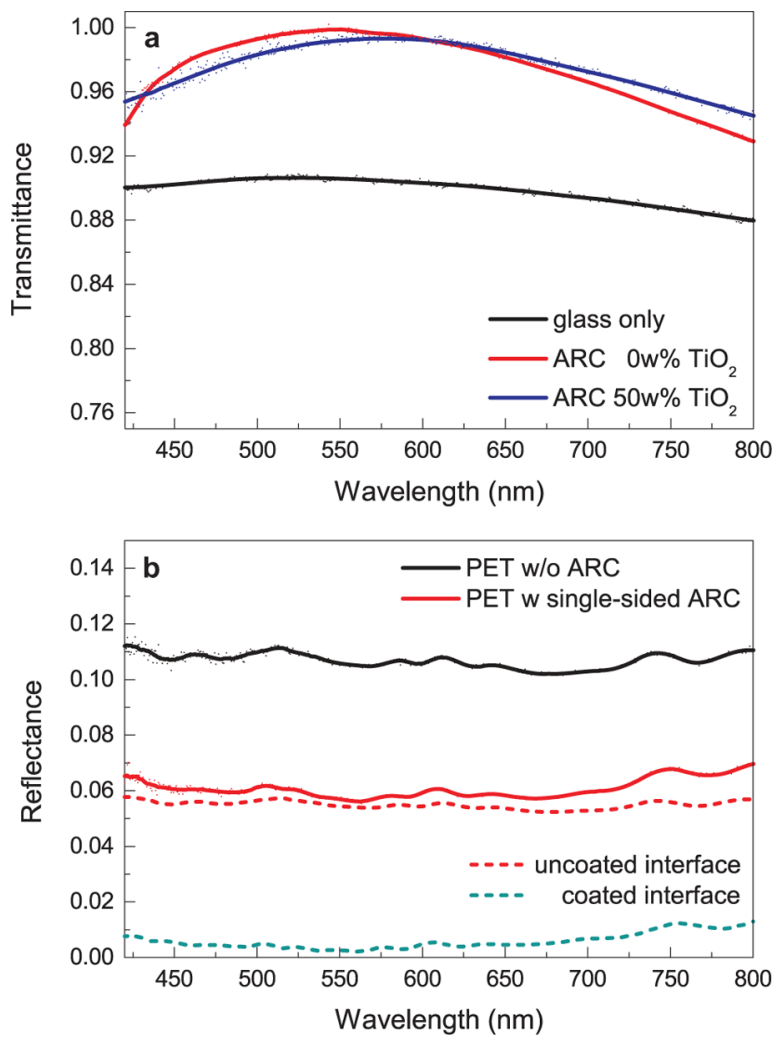

Figure 4. Optical properties of antireflective coatings. (a) Optical transmittance through a glass slide coated on both sides with an ARC containing 0 and 50 wt $\%$ photocatalytic $\mathrm{TiO}_{2}$ nanocrystals, respectively. (b) Reflectance of a flexible PET that was AR-coated on one side. The reflectance at the coated and uncoated interface can be derived by interfacial analysis based on experimental results comparing the foil coated on one side with the uncoated reference.

Information. While ARCs on PET and other flexible substrates have been demonstrated ${ }^{9,45}$ and photocatalytic self-cleaning on PET substrates has been shown, ${ }^{46}$ a solution of combining AR with photocatalysis does not exist.

The self-cleaning effect of photocatalytic $\mathrm{TiO}_{2}$ surfaces is based on the absorption of ultraviolet light in the $\mathrm{TiO}_{2}$. Some of the photoexcited charge carriers react with atmospheric oxygen to form radicals, which degrade nearby organic molecules. This so-called "cold combustion" mechanism enables self-cleaning through the conversion of organic pollutants to carbon dioxide, water, and mineral acids. ${ }^{27}$ As $\mathrm{TiO}_{2}$ only absorbs in the spectral range $\lambda<375 \mathrm{~nm}\left(E_{\text {gap }} \sim 3.3\right.$ $\mathrm{eV}^{28}$ ), it is important to closely mimic solar irradiation (AM 1.5 ) in the ultraviolet frequency range. This was achieved by a xenon lamp that was calibrated to match the intensity of ambient solar power in this spectral window (see Supporting Information).

The high photocatalytic efficiency of a BCP-34-derived ARC containing $50 \% \mathrm{TiO}_{2}$ is qualitatively demonstrated in Figure 5a. A fingerprint applied to the surface completely disappeared after AM 1.5 irradiation for $2 \mathrm{~h}$. Quantitative studies often use stearic acid, which readily assembles in a homogeneous layer onto inorganic surfaces, as an organic marker to monitor the photocatalytic performance of self-cleaning surfaces. ${ }^{27,47,48}$ Its decomposition can be monitored by Fourier transform infrared spectroscopy (FTIR).

Figure $5 \mathrm{~b}$ shows the decomposition of stearic acid adsorbed on a BCP-34-derived ARC containing $50 \% \mathrm{TiO}_{2}$. The 

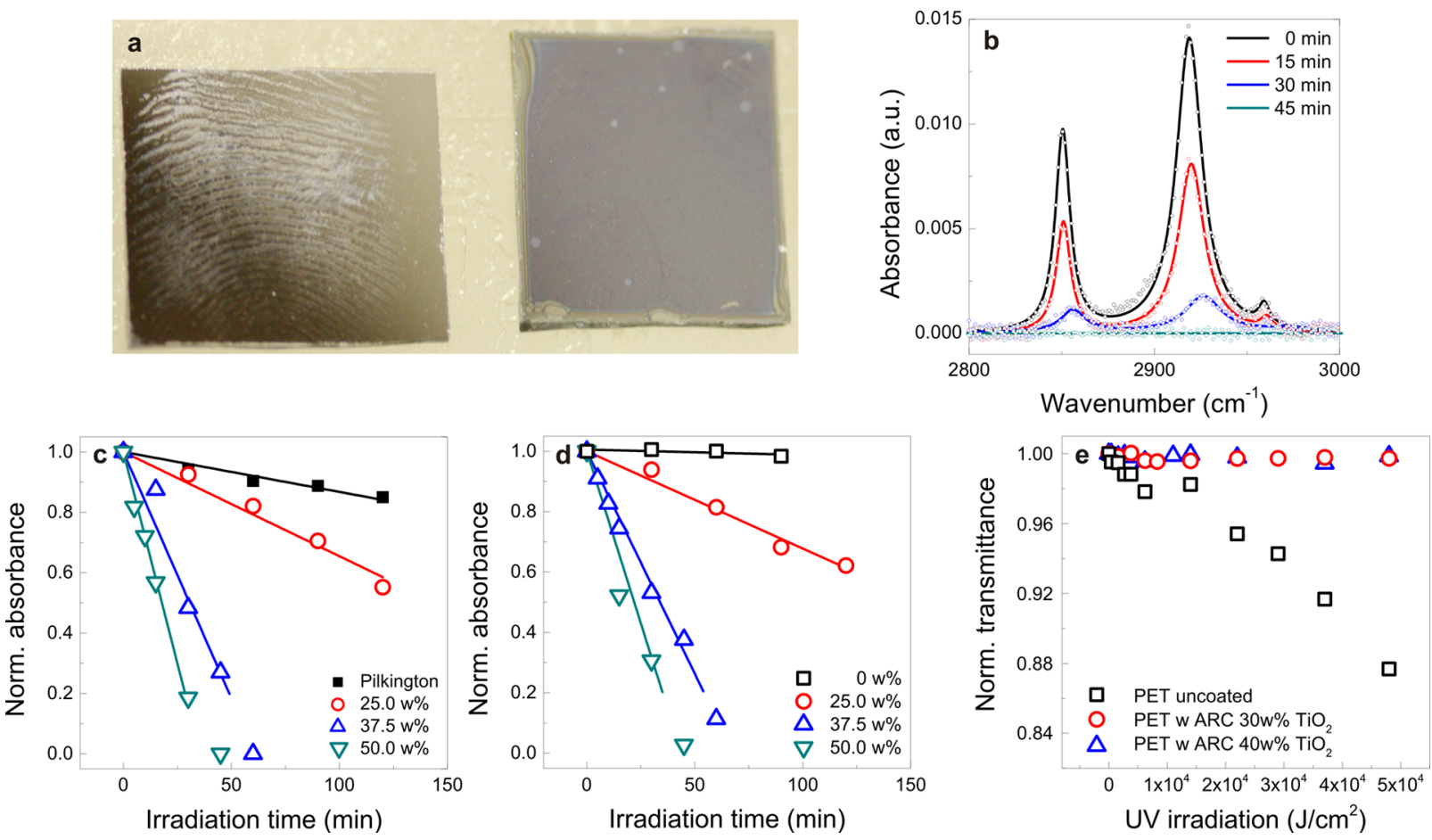

Figure 5. Self-cleaning ARCs. (a) Photodegradation of fingerprints applied on silicon substrates. While the fingerprint on a bare wafer (left) remained visible, it disappeared completely from a BCP-34 derived ARC containing 50\% $\mathrm{TiO}_{2}$ after irradiation with AM 1.5 light for 120 min (right). (b) Decomposition of stearic acid as a function of time adsorbed on a similar sample as in panel a (right). (c-d) Integrated peak area of stearic acid FTIR absorbance as a function of irradiation time for panel c, BCP-34 (pore size $\approx 33 \mathrm{~nm}$ ) and panel d, BCP-92 (pore size $\approx 53 \mathrm{~nm}$ ) derived ARCs. The full squares in panel $\mathrm{c}$ represent the commercial nonantireflective Pilkington Active glass, and the open squares in panel $\mathrm{d}$ are from a silica network that does not contain $\mathrm{TiO}_{2}$. (e) Degradation of initial transmittance upon UV irradiation, comparing ARC-30 wt \% $\mathrm{TiO} 2$ and ARC-40 wt $\%$ $\mathrm{TiO}_{2}$ coated sample with an uncoated reference. The annual UV irradiation in Central Europe is ${ }^{49} \sim 22000 \mathrm{~J} / \mathrm{cm}^{2}$.

integrated 2923 and $2853 \mathrm{~cm}^{-1}$ FTIR absorption peaks, stemming from symmetric and asymmetric $\mathrm{C}-\mathrm{H}$ stretching of $\mathrm{CH}_{2}$, respectively, were monitored as a measure of the stearic acid stability on the ARC surfaces. A rapid decay with irradiation time is apparent (see also Figure S7 in the Supporting Information). The decrease in stearic acid absorbance versus irradiation time is shown in Figure 5c,d for all samples. The intensity decrease can be fitted by a linear decay, and thus exhibits zeroth-order reaction characteristics. Samples derived from BCP-34 had rate constants of 0.36, 3.71, and $5.10 \times 10^{13}$ molecules/min for 25.0, 37.5, and $50.0 \mathrm{wt} \%$ $\mathrm{TiO}_{2}$ loading, respectively. ARCs with larger pores exhibited rate constants of $0.36,1.54$, and $3.12 \times 10^{13}$ molecules $/ \mathrm{min}$ for the three loading percentages.

Two clear trends emerge. First, increased $\mathrm{TiO}_{2}$ loading led to accelerated stearic acid decomposition in both sample series. Second, ARCs with smaller pores $(\sim 33 \mathrm{~nm})$ outperformed ARCs with larger pores $(\sim 53 \mathrm{~nm})$. This behavior can be linked to the random distribution of $\mathrm{TiO}_{2}$ nanocrystals in the silica matrix of Figure 3. Both, an increase in $\mathrm{TiO}_{2}$ loading and a reduction in strut diameter, lead to an increase in the number of nanocrystals that are located close to the strut surface and are therefore photocatalytically active. An overview of the photocatalytic decomposition rates is shown in the Supporting Information.

Stearic acid decomposition under solar irradiation was also investigated for pure silica ARCs. No decrease in FTIR absorbance was observed after $3 \mathrm{~h}$ of irradiation, indicating that stearic acid decomposition arises exclusively from the photocatalytic activity of the $\mathrm{TiO}_{2}$ nanocrystals. Commercial self- cleaning (but reflection-enhancing) Pilkington "Activ" glass showed a much weaker photocatalytic degradation of stearic acid with a reaction rate constant of $0.15 \times 10^{13}$ molecules $/ \mathrm{min}$.

The deposition of self-cleaning ARCs on flexible PET in lowcost, large area roll-to-roll fabrication techniques ${ }^{44}$ is promising, but long-term stability and robustness often limit their usefulness in commercial applications. In particular for outdoor applications (e.g., photovoltaics), UV-degradation of PET has been a core challenge. Commercially available UV-stabilized foils rely on bulk doping with UV-absorbers, which has limitations in thermal stability and dopant spectral matching. Ideally, UV light should be absorbed by a coating rather than entering the plastic substrate.

UV-stability tests were carried out by exposure of ARCcovered PET to UV irradiation of $48000 \mathrm{~J} / \mathrm{cm}^{2}$ in the $315-405$ $\mathrm{nm}$ spectral range, corresponding to a mean UV exposure of 26 months in Central Europe. Figure $5 \mathrm{e}$ and Supporting Information Figure S9 show that the transparency of ARCcoated films remained stable, while an uncoated reference showed a degradation in optical properties by over $12 \%$. This is a strong evidence that these ARCs are effective not only in terms of their antireflection and self-cleaning properties but also provide long-term UV protection.

A further important aspect is robustness against mechanical impact. ${ }^{50}$ Scratch tests comparing ARC-glass substrates with the only commercially available reference, where self-cleaning is based on superhydrophobicity and not photocatalysis, are shown in the Supporting Information. Our prototypes outperformed the commercially reference, maintaining the self-cleaning effect, which is lost in the case of super- 
hydrophobic coatings upon surface damage. On PET substrates, the ARC acts as a protective coating against mechanical impact, increasing the critical force for surface damage by a factor of 2.5 .

In conclusion, we report the manufacture of a highly porous ARC with high $\mathrm{TiO}_{2}$-nanoparticle loading that combines excellent optical antireflectivity with efficient photocatalytic activity. Our strategy relies on the assembly of high molecular weight BCP micelles that assemble into a sacrificial opal-type structure. After solidification by a sol-gel condensation reaction, this gives rise to a silica network with porosities of up to $73 \%$. This enabled the addition of high weight fractions of $\mathrm{TiO}_{2}$ nanocrystals without compromising the refractive index required for high performance ARCs. The combined AR and photocatalytic properties should substantially improve the longterm optical properties of porous ARCs, or even remove applied contaminants such a fingerprints. The low-temperature ARC processing enables the coating of flexible plastic substrates, paving the way for low-cost large area ARC manufacture.

\section{ASSOCIATED CONTENT}

\section{S Supporting Information}

Detailed experimental methods and supplementary figures. This material is available free of charge via the Internet at http://pubs.acs.org.

\section{AUTHOR INFORMATION}

\section{Corresponding Author}

*E-mail: u.steiner@phy.cam.ac.uk.

\section{Present Address}

${ }^{\perp}$ Institute of Materials Science, École Polytechnique Fédérale de Lausanne, 1015 Lausanne, Switzerland.

\section{Notes}

The authors declare no competing financial interest.

\section{ACKNOWLEDGMENTS}

This publication is based on work supported in part by Award No. KUS-C1-018-02 made by King Abdullah University of Science and Technology (KAUST), the European Community's Seventh Framework Programme (FP7/2007-2013) under Grant Agreement 246124 of the SANS project, the EPSRC (EP/F056702/1 and EP/F065884/1), the Department of Energy (DE-FG02 87ER45298) through the Cornell Fuel Cell Institute (CFCI) and the National Science Foundation (DMR-1104773). S.G. is grateful for support by the German National Academy of Sciences Leopoldina, Fellowship LPDS2012-13. M.S. is grateful to the University of South Carolina for startup funds. The authors greatly appreciate the invaluable help of Dr. Benjamin Schmidt-Hansberg and Dr. Mathias Kolle.

\section{REFERENCES}

(1) Macleod, H. Thin film optical filters, 3rd ed.; Institute of Physics Publishing: Bristol, 2001.

(2) Bruggeman, D. Ann. Phys. 1935, 416, 636.

(3) Hutchinson, N. J.; Coquil, T.; Navid, A.; Pilon, L. Thin Solid Films 2010, 518, 2141-2146.

(4) Walheim, S.; Schäffer, E.; Mlynek, J.; Steiner, U. Science 1999, $283,520-522$.

(5) Kim, S.; Cho, J.; Char, K. Langmuir 2007, 23, 6737-43.

(6) Thomas, I. M. Appl. Opt. 1992, 31, 6145-9.
(7) Lee, D.; Rubner, M. F.; Cohen, R. E. Nano Lett. 2006, 6, 230512.

(8) Hattori, H. Adv. Mater. 2001, 13, 51-54.

(9) Du, Y.; Luna, L. E.; Tan, W. S.; Rubner, M. F.; Cohen, R. E. ACS Nano 2010, 4, 4308-4316.

(10) Moghal, J.; Kobler, J.; Sauer, J.; Best, J.; Gardener, M.; Watt, A. A. R.; Wakefield, G. ACS Appl. Mater. Interfaces 2012, 4, 854-859.

(11) Hiller, J.; Mendelsohn, J.; Rubner, M. Nat. Mater. 2002, 1, 5963.

(12) Xi, J.-Q.; Schubert, M. F.; Kim, J. K.; Schubert, E. F.; Chen, M.; Lin, S.-Y.; Liu, W.; Smart, J. A. Nat. Photonics 2007, 1, 176-179.

(13) Wilson, S.; Hutley, M. Opt. Acta 1982, 29, 993-1009.

(14) Clapham, P.; Hutley, M. Nature 1973, 244, 281-282.

(15) Gombert, A.; Glaubitt, W.; Rose, K.; Dreibholz, J.; Bläsi, B.; Heinzel, A.; Sporn, D.; Döll, W.; Wittwer, V. Thin Solid Films 1999, $351,73-78$.

(16) Lohmueller, T.; Helgert, M.; Sundermann, M.; Brunner, R.; Spatz, J. P. Nano Lett. 2008, 8, 1429-1433.

(17) Li, Y.; Zhang, J.; Zhu, S.; Dong, H.; Jia, F.; Wang, Z.; Sun, Z.; Zhang, L.; Li, Y.; Li, H.; Xu, W.; Yang, B. Adv. Mater. 2009, 21, 47314734.

(18) Li, Y.; Zhang, J.; Yang, B. Nano Today 2010, 5, 117-127.

(19) Raut, H. K.; Ganesh, V. A.; Nair, A. S.; Ramakrishna, S. Energy Environ. Sci. 2011, 4, 3779-3804.

(20) Askar, K.; Phillips, B. M.; Fanga, Y.; Choi, B.; Gozubenli, N.; Jiang, P.; Jiang, B. Colloids Surf., A 2013, DOI: 10.1016/j.colsurfa.2013.03.004.

(21) Min, W.-L.; Jiang, B.; Jiang, P. Adv. Mater. 2008, 20, 39143918.

(22) Li, X.; Du, X.; He, J. Langmuir 2010, 26, 13528-13534.

(23) Park, K.-C.; Choi, H. J.; Chang, C.-H.; Cohen, R. E.; McKinley, G. H.; Barbastathis, G. ACS Nano 2012, 6, 3789-3799.

(24) Manca, M.; Cannavale, A.; De Marco, L.; Arico, A. S.; Cingolani, R; Gigli, G. Langmuir 2009, 25, 6357-6362.

(25) Yildirim, A.; Khudiyev, T.; Daglar, B.; Budunoglu, H.; Okyay, A. K.; Bayindir, M. ACS Appl. Mater. Interfaces 2013, 5, 853-860.

(26) Camargo, K. C.; Michels, A. F.; Rodembusch, F. S.; Kuhn, M. F.; Horowitz, F. Opt. Mater. Express 2012, 2, 969-977.

(27) Parkin, I. P.; Palgrave, R. G. J. Mater. Chem. 2005, 15, 16891695.

(28) Tang, H.; Berger, H.; Schmid, P.; F., L. Solid State Commun. 1994, 92, 267-271.

(29) Zhang, X.-T.; Sato, O.; Taguchi, M.; Einaga, Y.; Murakami, T.; Fujishima, A. Chem. Mater. 2005, 17, 696-700.

(30) Li, X.; He, J. ACS Appl. Mater. Interfaces 2013, 5, 5282-5290.

(31) Zhang, X.; Fujishima, A.; Jin, M.; Emeline, A. V.; Murakami, T. J. Phys. Chem. B 2006, 110, 25142-25148.

(32) Liu, Z.; Zhang, X.; Murakami, T.; Fujishima, A. Sol. Energy Mater. Sol. Cells 2008, 92, 1434-1438.

(33) Faustini, M.; Nicole, L.; Boissiere, C.; Innocenzi, P.; Sanchez, C.; Grosso, D. Chem. Mater. 2010, 22, 4406-4413.

(34) Templin, M.; Franck, A.; Chesne, A. D.; Leist, H.; Zhang, Y.; Ulrich, R.; Schädler, V.; Wiesner, U. Science 1997, 278, 1795-1798.

(35) Garcia, B.; Kamperman, M.; Ulrich, R.; Jain, A.; Gruner, S.; Wiesner, U. Chem. Mater. 2009, 21, 5397-5405.

(36) Niederberger, M.; Bartl, M.; Stucky, G. Chem. Mater. 2002, 14, 4364-4370.

(37) Kohn, P.; Pathak, S. K.; Stefik, M.; Ducati, C.; Wiesner, U.; Steiner, U.; Guldin, S. Nanoscale 2013, 5, 10518-10524.

(38) Guldin, S.; Hüttner, S.; Tiwana, P.; Orilall, M.; Ülgüt, B.; Stefik, M.; Docampo, P.; Kolle, M.; Divitini, G.; Ducati, C.; Redfern, S.; Snaith, H.; Wiesner, U.; Eder, D.; Steiner, U. Energy Environ Sci 2011, 4, 225-233.

(39) Guldin, S.; Kolle, M.; Stefik, M.; Langford, R.; Eder, D.; Wiesner, U.; Steiner, U. Adv. Mater. 2011, 23, 3664-3668.

(40) Rubinstein, M.; Colby, R. H. Polymer Physics Oxford University Press, 2003, 1st edition.

(41) Jaeger, H.; Nagel, S. Science 1992, 255, 1523-1531. 
(42) Lopez-Lopez, C.; Colodrero, S.; Raga, S.; Lindstrom, H.; Fabregat-Santiago, F.; Bisquert, J.; Miguez, H. J. Mater. Chem. 2012, 22, 1751-1757.

(43) Hsueh, H.-Y.; Chen, H.-Y.; She, M.-S.; Chen, C.-K.; Ho, R.-M.; Gwo, S.; Hasegawa, H.; Thomas, E. L. Nano Lett. 2010, 10, 49945000.

(44) Krebs, F. C. Sol. Energy Mat. Sol. Cells 2009, 93, 394-412.

(45) Kum, B. G.; Park, Y. C.; Chang, Y. J.; Jeon, J. Y.; Jang, H. M. Thin Solid Films 2011, 519, 3778-3781.

(46) Nakata, K.; Sakai, M.; Ochiai, T.; Murakami, T.; Takagi, K.; Fujishima, A. Langmuir 2011, 27, 3275-3278.

(47) Paz, Y.; Luo, Z.; Rabenberg, L.; Heller, A. J. Mater. Res. 1995, 10, $2842-2848$.

(48) Mills, A.; Lepre, A.; Elliott, N.; Bhopal, S.; Parkin, I. P.; ONeill, S. J. Photochem. Photobiol., A 2003, 160, 213-224.

(49) Mines ParisTech; SODA, Mean of Daily Irradiation in UV in the World. http://www.soda-is.com, 2008 (accessed June 18, 2013).

(50) Yao, L.; He, J. Langmuir 2013, 29, 3089-3096. 